J.Edu. Sci , Vol. (19) No.(3) 2007

\title{
The Lower Bounds of Eight and Fourth Blocking Sets and Existence of Minimal Blocking Sets
}

\author{
Abdul Khalik,L.Yasin \\ Dept. of Math \\ Nada Yassen Kasm Yahya \\ Dept. of Math. \\ College of Computers Sciences and Math. College of Education \\ University of Mosul
}

Received
29/11/2006

Accepted

21/2/2007

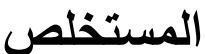

في هذا البحث حصلنا على نتيجنين رئيسيتين متعلقتين بحجم المجموعة القالبية من

النمط-8والنمط-4 في المستوي (PG(2,16. وتمثلت النتيجتان في إيجادنا قوسا تاما جديدا -

(k,13) - موجود (129,9) لم يسبق الحصول عليه في البحوث الحديثة، وإثباتتا أن القوس التام

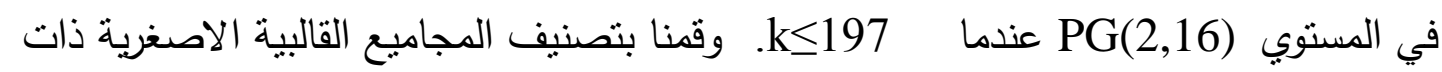

الحجم 8 في المستوي الاسقاطي PG(2,4 ، وأثبتتا أن المجموعة الاصغرية ذات الحجم 8 هي

من النوع Rédei -type وأعطينا بعض خواص المجموعة القالبية الاصغرية ذات الحجم 10

في المستوي الاسقاطي(PG(2,5 ، وحصلنا على مثال للمجموعة القالبية الاصغرية ذات الحجم 10 ذات قاطع رباعي على الأكثر في المستوي الاسقاطي PG(2,5 وأثبتنا أن المجموعة Rédei -type الاصغرية ذات الحجم 10 هي من النوب الني
\end{abstract}

\section{ABSTRACT}

This paper contains two main results relating to the size of eight and fourth blocking set in PG(2,16). First gives new example for $(129,9)$ complete arc. The second result we prove that there exists $(\mathrm{k}, 13)$ complete arc in $\operatorname{PG}(2,16), \mathrm{k} \leq 197$. We classify the minimal blocking sets of size eight in PG $(2,4)$.We show that Rédei -type minimal blocking sets of size eight exist in $\mathrm{PG}(2,4)$. Also we classify the minimal blocking sets of size ten in $\operatorname{PG}(2,5)$, We obtain an example of a minimal blocking set of size ten with at most 4-secants.We show that Rédei type minimal blocking sets of size ten exists in $\mathrm{PG}(2,5)$.

ملاحظة:البحث مستل من الأطروحة 


\section{The Lower Bounds of Eight and Fourth ...}

\subsection{Introduction:}

$A(k, n)$-arc $K$ in $P G(2, q)$ is a set of $k$ points such that there is some $\mathrm{n}$ but no $\mathrm{n}+1$ of them are collinear. $\mathrm{A}(\mathrm{k}, \mathrm{n})$-arc $\mathrm{K}$ is complete if there is no $(\mathrm{k}+1, \mathrm{n})$-arc containing it. The maximum value of $\mathrm{k}$ which a $(\mathrm{k}, \mathrm{n})$-arc $\mathrm{K}$ exist in $\mathrm{PG}(2, \mathrm{q})$ will be denoted by $\mathrm{m}(\mathrm{n})_{2, \mathrm{q}}[6]$.

A t-fold blocking set $\mathrm{B}$ in a projective plane, is a set of points such that each line contains at least $t$ points of $B$ and some line contains exactly $\mathrm{t}$ points of $\mathrm{B}[1]$. For $\mathrm{t}=1$, $\mathrm{a} 1$-fold blocking set is called a blocking set. A trivial blocking set $\mathrm{B}$ is a blocking set containing a line of $\mathrm{PG}(2, \mathrm{q})$. A t- blocking set is called minimal (irreducible)when no proper subset of it is a t- blocking set [12]. For $t=2,3,4, \ldots$ then $t$ - blocking set is called respectively double blocking set, triple blocking set, fourth blocking set...etc. $(\mathrm{k}, \mathrm{n})$-arcs and $\mathrm{t}$ - blocking sets are in fact just complements of each other in a projective plane, with $\mathrm{n}+\mathrm{t}=\mathrm{q}+1$.

Richardson was the first one to look at larger planes [11]. He showed that the minimal size of a blocking set in $\operatorname{PG}(2,3)$ is 6 , and noted that Baer subplanes are examples of blocking sets of size $\mathrm{q}+\sqrt{q}+1$ in projective planes of square order. After that things were quiet for 13 years until Di paola[4] introduced the idea of a projective triangle, which gives an example of a blocking set of size $3(q+1) / 2$ in Desargusian planes of odd order. That projective triangles exist in these planes was shown by Bruen, who also obtained the general lower bound $\mathrm{q}+\sqrt{q}+1$ for the size of a blocking set in arbitrary projective plane of odd order q.

Further results obtained by Bruen [3], giving the upper bound $\mathrm{q}^{\sqrt{q}}+1$ for a minimal blocking set in any projective plane of order $\mathrm{q}$, and make the connection with Re'dei 's work on lacunary polynomials[ 10 ]. The fundamental results are for the structure of blocking sets however was only realized much later and in this course the emphasis will be to explain in some detail the recent developments and the connection between Re'dei 's work on lacunary polynomials and small blocking sets and multiple blocking sets in Desargusian projective planes.

\subsection{The projective plane $\operatorname{PG}(2,16)$ :}

Let $f(x)=x^{3}+x^{2}+x+\lambda$ be a monic polynomial over $G F(16)$ then companion matrix of $\mathrm{f}(\mathrm{x})$

$$
\mathrm{T}=\left[\begin{array}{lll}
0 & 1 & 0 \\
0 & 0 & 1 \\
\lambda & 1 & 1
\end{array}\right]
$$

is cyclic projectivety on $\mathrm{PG}(2,16)$. Note that in $\mathrm{PG}(2,16),\{\lambda=-\lambda\}$ 
Let $\pi=\mathrm{Gf}(16)=\left\{0,1, \lambda^{\mathrm{i}}: \mathrm{i} \in \mathrm{N}_{14}: \lambda^{15}=1\right\}$

We write the elements of $\pi$ as $1,2,3 \ldots, 16$ instead of $0,1, \lambda, \ldots, \lambda^{14}$, respectively. So the cyclic projectivety becomes:

$$
\mathrm{T}=\left[\begin{array}{lll}
1 & 2 & 1 \\
1 & 1 & 2 \\
3 & 2 & 2
\end{array}\right]
$$

The number of point in the $\mathrm{PG}(2,16)$ has 273 points and 273lines and every line passes throw 17 points.

Let $\mathrm{p}_{0}$ be the point $\mathrm{U}_{0}=(2,1,1)$ then $\mathrm{Pi}=\mathrm{P}_{0} \mathrm{~T}^{\mathrm{i}}, \mathrm{i}=0, \ldots, 272$, are the 273 points of $\mathrm{PG}(2,16)$. See $[8$, Table(1)]

Let $\mathrm{L}_{1}$ be the line which contains the points $0,1,4,16,26,57,64,91,93,99,104,123,143,205,219,228,256$, then $\mathrm{L}_{\mathrm{i}}=\mathrm{L}_{1} \mathrm{~T}^{\mathrm{i}-1}, \mathrm{i}=1, \ldots, 273$, are the lines of $\mathrm{PG}(2,16)$, the 273 lines $\mathrm{L}_{\mathrm{i}}$ are given by the rows in $[8$, Table(2)].

\subsection{Eight blocking sets in $\mathrm{PG}(2,16)$}

The object of this section is to obtain good lower bounds for the size of eight blocking sets in $\mathrm{PG}(2, \mathrm{q})$, q is square integer.

\section{Theorem( 2.1.1) ( $\mathrm{q}>9, \mathrm{q}$ is a square):}

Let $B$ be an eight blocking set in $\operatorname{PG}(2, q)$, $q$ is square such that through each of its points there are $\sqrt{ } \mathrm{q}+1$ lines, each lines contains at least $\sqrt{ } \mathrm{q}+8$ points of $\mathrm{B}$ and forming a dual Baer subline .Then

( 1 ) For $\mathrm{q}>64$, B has at least $8 \mathrm{q}+2 \sqrt{ } \mathrm{q}+8$ points.

( 2 ) For $q=16$, B has at least $8 q+\sqrt{ } q+10$ points.

Proof. ( 1 )Call the lines meeting $B$ in $\sqrt{ } \mathrm{q}+8$ or more points long lines . If two long lines meet out side of $B$, then $B$ has at least $2(\sqrt{ } q+8)+8(q-$ $1)=8 q+2 \sqrt{ } q+8$ points and the desired bound is obtained. Hence $|B| \geq 8 q+2 \sqrt{ } q+8$. So to assume that two long lines meet in $B$.Take 1 , a long line , and $\mathrm{p}$, a point of $\mathrm{B}$ not on $\mathrm{l}$. Then the long lines through $\mathrm{p}$ contain a dual Baer subline and meet 1 in a Baer subline. Let $Q$ be a point on this Baer subline. Consider long lines through a point on an 8 -secant to Q. These meet 1 in another Baer subline not containing Q. Two Baer sublines meet in at most two points and so 1 has at least $2 \sqrt{ } \mathrm{q}$ points. Since 1 was arbitrary every long line has at least $2 \sqrt{ } \mathrm{q}$ points and it follows that $B$ has at least $(\sqrt{ } q+1)(2 \sqrt{ } q-1)+1+7(q-\sqrt{ } q)=9 q-6 \sqrt{ } q$ points. Since $9 q-6 \sqrt{ } q \geq 8 q+2 \sqrt{q}+8$ so that $|B| \geq 8 q+2 \sqrt{q}+8$ points. 


\section{The Lower Bounds of Eight and Fourth ...}

Proof. ( 2 ) If two long lines meet out side of $B$, then $B$ has at least $2(\sqrt{ } \mathrm{q}+8)+8(\mathrm{q}-1)=8 \mathrm{q}+2 \sqrt{\mathrm{q}+8}$ points. Hence $|\mathrm{B}| \geq 8 \mathrm{q}+2 \sqrt{\mathrm{q}+8}$.

Let $p \in B$, through $B$, since there are $\sqrt{ } q+1$ long lines through $p$. $B$ has at least $(\sqrt{ } q+1)(\sqrt{ } q+7)+1+7(q+1-(\sqrt{ } q+1))=8 q+\sqrt{ } q+8$ points.

Now $|B| \geq 140$. If this bound is a chafed then (k,9)-arc has $k=133$ and that impossible .See Table(3)from [2]. If $|B|=8 q+\sqrt{ } q+9$ then $k=132$, that impossible. Since $k \leq 131$, hence $|B| \geq 8 q+\sqrt{ } q+10$.

Table (3)

The size of the largest $(\mathrm{k}, \mathrm{n})$-arc in $\mathrm{PG}(2, \mathrm{q})$ for small $\mathrm{q}$

\begin{tabular}{|c|c|c|c|c|c|c|c|c|c|c|c|}
\hline$n^{q}$ & 3 & 4 & 5 & 7 & 8 & 9 & 11 & 13 & $\overline{16}$ & $\overline{17}$ & 19 \\
\hline 2 & 4 & 6 & 6 & 8 & 10 & 10 & 12 & 14 & 18 & 18 & 20 \\
\hline 3 & & 9 & 11 & 15 & 15 & 17 & 21 & 23 & $28 \ldots 33$ & $28 \ldots 35$ & $31 \ldots 39$ \\
\hline 4 & & & 16 & 22 & 28 & 28 & $32 \ldots 34$ & $38 \ldots 40$ & 52 & $48 \ldots 52$ & $\overline{52 \ldots 58}$ \\
\hline 5 & & & & 29 & 33 & 37 & $43 \ldots 45$ & $49 \ldots 53$ & 65 & $61 \ldots 69$ & $68 \ldots 77$ \\
\hline 6 & & & & 36 & 42 & 48 & 56 & 64..66 & $78 \ldots 82$ & $78 \ldots 86$ & $86 \ldots 96$ \\
\hline 7 & & & & & 49 & 55 & 67 & 79 & $993 \ldots 97$ & $994 \ldots 103$ & $105 \ldots 115$ \\
\hline 8 & & & & & & 65 & $77 \ldots 78$ & 92 & 120 & $114 \ldots 120$ & $124 \ldots 134$ \\
\hline 9 & & & & & & & $89 \ldots 90$ & 105 & $128 \ldots 131$ & 137 & $147 \ldots 153$ \\
\hline 10 & & & & & & & $100 \ldots 102$ & $118 \ldots 119$ & $142 \ldots 148$ & 154 & 172 \\
\hline 11 & & & & & & & & $132 \ldots 133$ & $159 \ldots 164$ & $166 \ldots 171$ & 191 \\
\hline 12 & & & & & & & & $145 \ldots 147$ & $180 \ldots 181$ & $182 \ldots 189$ & $204 \ldots 210$ \\
\hline 13 & & & & & & & & & $195 \ldots 199$ & $204 \ldots 207$ & $225 \ldots .230$ \\
\hline 14 & & & & & & & & & $210 \ldots 214$ & $221 \ldots 225$ & $242 \ldots 250$ \\
\hline 15 & & & & & & & & & 231 & $239 \ldots 243$ & $262 \ldots 271$ \\
\hline 16 & & & & & & & & & & $256 \ldots 261$ & $285 \ldots 290$ \\
\hline 17 & & & & & & & & & & & $305 \ldots 311$ \\
\hline 18 & & & & & & & & & & & $324 \ldots 330$ \\
\hline
\end{tabular}

\section{Corollary (2.1.2):}

There exists $(129,9)$-arc in $\mathrm{PG}(2,16)$.

Proof. Finding a maximum(k,9)-arc is equivalent to finding the minimum eight blocking sets by considering complements .

Theorem(2.1.1 )gives lower Bound for eight blocking set with $8 q+2 \sqrt{ } q+8$, if two lines with $\sqrt{ } q+8$ points intersect outside of the eight blocking set. Eight blocking set must have at least 144 points there were eight blocking sets exactly 144 points, and equivalently a $(129,9)$-arc does exist Example(2.1.4). Hence $\mathrm{k}=129$ is a new sharp upper bound for $(\mathrm{k}, 9)$-arc. See Table(3). 


\subsubsection{The value of $\mathrm{m}(\mathrm{n})_{2, \mathrm{q}}$}

In this section example of large $(\mathrm{k}, \mathrm{n})$-arcs in $\mathrm{PG}(2,16)$ are given. Improvements on the upper bounds of $\mathrm{m}(\mathrm{n})_{2,16}$ obtained from Corollary (2.1.2) are made . Example(2.1.4) constructed by taking random subsets of the internal points of a conic.

Example(2.1.4): The set of the following points

$$
\begin{aligned}
& \left\{(1,0,0),(0,1,0),(0,0,1),(1,1,1),\left(1, \lambda^{6}, \lambda^{9}\right),\left(1, \lambda^{8}, \lambda^{7}\right),\left(1, \lambda^{10}, \lambda^{5}\right),\left(1, \lambda^{5}, \lambda^{10}\right)\right. \\
& ,\left(1, \lambda, \lambda^{14}\right),\left(1, \lambda^{7}, \lambda^{8}\right),\left(1, \lambda^{12}, \lambda^{3}\right),\left(1, \lambda^{2}, \lambda^{13}\right),\left(1, \lambda^{3}, \lambda^{12}\right),\left(1, \lambda^{13}, \lambda^{2}\right) \\
& ,\left(1, \lambda^{14}, \lambda\right),\left(1, \lambda^{9}, \lambda^{6}\right),\left(1, \lambda^{4}, \lambda^{11}\right),\left(1, \lambda^{11}, \lambda^{4}\right),\left(1, \lambda^{8}, \lambda^{6}\right),\left(1, \lambda^{14}, \lambda^{14}\right) \\
& ,\left(1, \lambda^{7}, \lambda^{2}\right),\left(1, \lambda^{9}, \lambda\right),\left(1, \lambda^{3}, \lambda^{8}\right),\left(1, \lambda^{11}, \lambda^{5}\right),\left(1, \lambda^{10}, \lambda^{13}\right),\left(1, \lambda^{5}, \lambda^{9}\right) \\
& ,\left(1,0, \lambda^{7}\right),\left(0,1, \lambda^{8}\right),\left(1, \lambda^{13}, \lambda^{4}\right),\left(1, \lambda^{6}, 0\right),\left(1,1, \lambda^{10}\right),\left(1, \lambda^{14}, \lambda^{13}\right), \\
& \left(1, \lambda^{13}, 1\right),\left(1, \lambda^{3}, \lambda^{14}\right),\left(1, \lambda^{8}, \lambda^{9}\right),\left(1, \lambda^{6}, \lambda^{3}\right),\left(1, \lambda, \lambda^{8}\right),\left(1, \lambda^{10}, \lambda^{6}\right),\left(1, \lambda^{4}, \lambda^{12}\right), \\
& \left(1, \lambda^{9}, \lambda^{7}\right),(1,1, \lambda),\left(1, \lambda^{7}, \lambda^{4}\right),\left(1,1, \lambda^{3}\right),\left(0,1, \lambda^{9}\right),(1, \lambda, 1),\left(1,0, \lambda^{13}\right) \\
& ,\left(1, \lambda^{2}, \lambda^{7}\right),\left(1, \lambda^{12}, 1\right),\left(1, \lambda^{9}, \lambda^{11}\right),\left(1, \lambda, \lambda^{5}\right),\left(1, \lambda^{11}, \lambda^{12}\right),\left(1, \lambda^{2}, \lambda\right),\left(1, \lambda^{5}, \lambda^{11}\right) \\
& ,\left(1, \lambda^{12}, 0\right),\left(0,1, \lambda^{7}\right),\left(1, \lambda^{10}, 12\right),\left(1, \lambda^{5}, \lambda^{5}\right),\left(1, \lambda^{4}, \lambda^{2}\right),\left(1, \lambda^{7}, \lambda^{3}\right), \\
& \left(1, \lambda^{4}, \lambda^{9}\right),\left(1,1, \lambda^{12}\right),\left(1, \lambda^{13}, \lambda\right),\left(1,0, \lambda^{5}\right),\left(0,1, \lambda^{4}\right),\left(1, \lambda, \lambda^{11}\right),\left(1, \lambda^{12}, \lambda^{12}\right), \\
& \left(1, \lambda^{8}, \lambda^{4}\right),\left(1, \lambda^{6}, \lambda^{7}\right),\left(1, \lambda^{14}, \lambda^{9}\right),\left(1, \lambda^{12}, \lambda^{2}\right),\left(1, \lambda^{4}, 0\right),\left(1, \lambda^{11}, \lambda^{13}\right), \\
& \left(1, \lambda^{2}, \lambda^{6}\right),\left(1, \lambda^{8}, 1\right),\left(1, \lambda^{10}, 0\right),\left(1, \lambda^{7}, \lambda^{5}\right),\left(1,0, \lambda^{2}\right),\left(1, \lambda^{2}, \lambda^{8}\right), \\
& \left(1, \lambda^{6}, \lambda^{12}\right),\left(1, \lambda^{11}, \lambda^{11}\right),\left(1,0, \lambda^{9}\right),\left(1, \lambda^{5}, \lambda^{6}\right),\left(0,1, \lambda^{13}\right),\left(1, \lambda^{2}, \lambda^{14}\right), \\
& \left(1, \lambda^{5}, \lambda\right),\left(1,1, \lambda^{4}\right),\left(1, \lambda^{12}, \lambda^{4}\right),\left(1, \lambda^{6}, \lambda^{8}\right),\left(0,1, \lambda^{6}\right),\left(1, \lambda^{8}, \lambda^{3}\right), \\
& \left(1, \lambda, \lambda^{9}\right),\left(1, \lambda^{4}, \lambda^{7}\right),\left(1, \lambda^{14}, \lambda^{10}\right),\left(1,0, \lambda^{14}\right),\left(1, \lambda^{9}, \lambda^{14}\right),\left(1, \lambda^{2}, \lambda^{2}\right), \\
& \left(1, \lambda^{13}, \lambda^{10}\right),\left(1, \lambda^{7}, \lambda^{13}\right),\left(1,1, \lambda^{5}\right),\left(1, \lambda^{6}, \lambda^{13}\right),\left(1, \lambda^{8}, \lambda^{12}\right),\left(1, \lambda, \lambda^{10}\right), \\
& \left(1, \lambda^{13}, \lambda^{6}\right),\left(1, \lambda^{10}, \lambda^{11}\right),\left(1, \lambda^{8}, \lambda\right),\left(1, \lambda^{5}, \lambda^{3}\right),\left(1, \lambda^{4}, \lambda^{3}\right) \\
& \left(1, \lambda^{12}, \lambda^{11}\right),\left(1, \lambda^{6}, 1\right),\left(1, \lambda^{9}, \lambda^{5}\right),\left(1, \lambda^{10}, \lambda^{4}\right),\left(1, \lambda^{7}, 1\right),\left(1, \lambda^{3}, \lambda^{8}\right), \\
& \left(1, \lambda^{14}, \lambda^{8}\right),\left(1, \lambda^{3}, \lambda^{7}\right),\left(1, \lambda^{3}, \lambda^{10}\right),\left(1, \lambda^{4}, \lambda^{6}\right),\left(1,1, \lambda^{9}\right),\left(1, \lambda, \lambda^{13}\right), \\
& \left(1, \lambda^{7}, 0\right),\left(0,1, \lambda^{2}\right),\left(1, \lambda^{3}, \lambda^{3}\right),\left(1, \lambda^{11}, \lambda^{14}\right),\left(1, \lambda^{9}, \lambda^{2}\right),\left(1, \lambda^{3}, \lambda\right) \\
& \left.,\left(1, \lambda^{11}, \lambda^{10}\right),\left(1, \lambda^{5}, 0\right),\left(1, \lambda^{14}, \lambda^{4}\right),\left(1,0, \lambda^{11}\right)\right\} \text {. Forms a }(129,9) \text {-arc } \\
& \text { in } \mathrm{PG}(2,16) \text { with secant distribution }
\end{aligned}
$$$$
\mathrm{T}_{0}=8, \mathrm{~T}_{1}=9, \mathrm{~T}_{2}=0, \mathrm{~T}_{3}=0, \mathrm{~T}_{4}=0, \mathrm{~T}_{5}=0, \mathrm{~T}_{6}=0, \mathrm{~T}_{7}=0, \mathrm{~T}_{8}=120 \text { and } \mathrm{T}_{9}=136 \text {. }
$$

\subsection{Fourth blocking sets in $\mathrm{PG}(2,16)$}

The object of this section is to obtain good lower bounds for the size of a fourth blocking sets in $\mathrm{PG}(2, \mathrm{q}), \mathrm{q}$ is square.

Theorem (2.2.1) ( $\mathrm{q}>9, \mathrm{q}$ is a square)

Let $B$ be a fourth blocking set in $P G(2, q)$, $q$ is square, such that through each of its points there are $\sqrt{ } \mathrm{q}+1$ lines, each containing at least $\sqrt{ } \mathrm{q}+4$ points of $\mathrm{B}$ and forming a dual Baer subline. Then $\mathrm{B}$ has at least $4 \mathrm{q}+2 \sqrt{\mathrm{q}+4}$ points.

Proof. Call the lines meeting $B$ in $\sqrt{ } \mathrm{q}+4$ or more points long lines .If two long lines meet out side of $\mathrm{B}$, then $\mathrm{B}$ has at least 


\section{The Lower Bounds of Eight and Fourth ...}

$2(\sqrt{ } q+4)+4(q-1)=4 q+2 \sqrt{ } q+4$ points and the desired bound is obtained .So assume that two long lines meet in $\mathrm{B}$. let 1 be a long line and $\mathrm{p}$ a point of $\mathrm{B}$ not on 1.Then the long lines through $\mathrm{p}$ contain a dual Baer subline and meet 1 in a Baer subline. Let $\mathrm{Q}$ be a point on this Baer subline. Consider long lines through a point on a 4-secant to $\mathrm{Q}$. These meet 1 in another Baer subline not containing Q. Two Baer subline meets in at most two points and so 1 has at least $2 \sqrt{q}$ points . Since 1 was arbitrary every long line has at least $2 \sqrt{\mathrm{q}}$ points and it follows that $\mathrm{B}$ has at least $(\sqrt{ } \mathrm{q}+1)(2 \sqrt{\mathrm{q}}-1)+1+3(\mathrm{q}-\sqrt{\mathrm{q}})=5 \mathrm{q}-2 \sqrt{\mathrm{q}}$ points.

For $\mathrm{q}>16$, $\mathrm{q}$ square $5 \mathrm{q}-2 \sqrt{\mathrm{q} \geq 4 \mathrm{q}}+2 \sqrt{\mathrm{q}+4}$. If $\mathrm{q}=16$ then $5 \mathrm{q}-2 \sqrt{\mathrm{q}=72}$ and $(\mathrm{k}, 13)$ has 201 points and that is impossible, see Table(3). Therefore $|\mathrm{B}| \geq 4 \mathrm{q}+2 \sqrt{\mathrm{q}}+4$.

Corollary (2.2.2):

There exists $(\mathrm{k}, 13)$-arc in $\mathrm{PG}(2,16), \mathrm{k} \leq 197$

Proof. Finding a maximum(k,13)-arc is equivalent to finding the minimum fourth blocking set by considering complements . Theorem (2.2.1)gives lower bound for fourth blocking set with $4 q+2 \sqrt{q}+4$. Fourth blocking set must have at least 76 points, so since $n=q+1-t$, then $(\mathrm{k}, 13)$-arcs have $\mathrm{k} \leq 197$.

3.1 On Blocking sets:

In this section we have given the following information on the structure of such blocking sets.

\section{Definition (3.1.1) ( unital):[3]}

Points, that every line $q \sqrt{q}+1$ A unital in $\mathrm{PG}(2, \mathrm{q})$ is a set $\mathrm{U}$ of $\sqrt{q}+1$ joining two points of $\mathrm{U}$ intersects $\mathrm{U}$ in precisely points.

again straight forward counting gives that all other lines of the plane there is intersect $U$ in precisely one point, and in fact at each point of $U$ a unique tangent. So a unital is a minimal blocking set. In fact it turns out to be the largest one.

Theorem(3.1.2) : [3]

let $\mathrm{B}$ be a minimal blocking set in $\operatorname{PG}(2, \mathrm{q})$. Then $|\mathrm{B}| \leq \mathrm{q} \sqrt{q}+1$ with equality if and only if $B$ is a unital in $\operatorname{PG}(2, q), q$ is square .

\section{Theorem (3.1.3):[7]}

In $\mathrm{PG}(2, \mathrm{q}), \mathrm{q}$ square, $\mathrm{q} \geq 25$ or $\mathrm{q}=9$, there is no minimal blocking set of size $q \sqrt{q}$. 
Theorem(3.1.4):[7]

For $\mathrm{q}$ square , $\mathrm{q} \geq 16$, there is no minimal blocking $\mathrm{k}$-set $\mathrm{B}$ $\leq \mathrm{k} \leq q+\sqrt{q}+1$ in $\mathrm{PG}(2, \mathrm{q})$ with $q+2 \sqrt{q}+1$

Theorem(3.1.5):[7]

In a Desargusian plane of order at least 4 there exists a blocking set of order $k$ if $2 q-1 \leq k \leq 3 q-3$.

\subsection{Minimal Blocking sets in PG(2,4):}

From now on, let $\mathrm{B}$ be a minimal blocking set of size eight in $\mathrm{PG}(2,4)$, since $\mathrm{B}$ is non-trivial a line 1 intersect $\mathrm{B}$ in at most four points.

Lemma (3.2.1): There's at most two 4-secants through any point of B. Proof. Every two 4-secant to B are intersect in a point on B.If two 4secants inters in $\mathrm{p} \quad \mathrm{B}$ then $|\mathrm{B}| \geq 2 * 4+3=11$, which is impossible, Assume there is a three 4-secant through a point $\mathrm{p} \in \mathrm{B}$, then $|\mathrm{B}| \geq 1+3 * 3=10$ and that is impossible. So through every point of B there is at most two 4-secants.

Lemma (3.2.2): If B has no 4- secants, then B has at least one secant with at least three points.

Proof. Suppose there are only 1-,2-secants, let the number of them be denoted by $\mathrm{a}$ and $\mathrm{b}$. Then the following equations must hold by standard counting arguments.

$$
\begin{aligned}
& a+b=21 \\
& a+2 b=40 \\
& 2 b=56 \ldots
\end{aligned}
$$

From equation(3), we get $b=28$ which is impossible.

Lemma (3.2.3): If B has no 3-secant, then B has at least one 4-secant. Proof. Suppose there are only 1-,2-, and 4- secants. let the number of them be denoted by a , b, $d$ recp. Then the following equations must hold by standard counting arguments.

$$
\begin{aligned}
& a+b+d=21 \\
& a+2 b+4 d=40 \\
& 2 b+12 d=56
\end{aligned}
$$

From these equations, we get $\mathrm{d}=3$. 


\section{The Lower Bounds of Eight and Fourth ...}

Theorem( 3.2.4) : Let B be a non-trivial blocking set . Let the number of 1-, 2-, 3- and 4-secants be denoted by a, b, c, d resp. Then we have one of the following possibilities:

\begin{tabular}{|c|c|c|c|c|}
\hline a & B & c & d & Possibilities \\
\hline 8 & 10 & 0 & 3 & (i ) \\
\hline 9 & 7 & 3 & 2 & ( ii ) \\
\hline 10 & 4 & 6 & 1 & (iii) \\
\hline 11 & 1 & 9 & 0 & ( iv ) \\
\hline
\end{tabular}

Proof. The standard counting arguments give:

$\mathrm{a}+\mathrm{b}+\mathrm{c}+\mathrm{d}=21 \quad \ldots$

$a+2 b+3 c+4 d=40 \ldots$

$2 b+6 c+12 d=56$

From these we can deduce

$\mathrm{a}=11-\mathrm{d}$;

$\mathrm{b}=1+3 \mathrm{~d}$;

$c=9-3 \mathrm{~d}$

Since $c \geq 0$, then $0 \leq \mathrm{d} \leq 3$.

We first show that first and fourth solution of Theorem (3.2.4) are not possible:

Theorem( 3.2.5):

The first solution $(8,10,0,3)$ and fourth solution $(11,1,9,0)$ of Theorem (3.2.4) do not exists.

Proof. (i)let $\mathrm{B}$ be a blocking set having the solution $(8,10,0,3)$, and assume $1_{1}, l_{2}, l_{3}$ be the three 4 -secants of $B$ : If $1_{1} \cap l_{2} \cap l_{3}=\{p\}$. Then $p$ must be in $\mathrm{B}$, and that contradicts $\operatorname{Lemma(3.2.1).~Now~if~} 1_{1}, 1_{2}, 1_{3}$ are triangular, so $|B| \geq 9$ and that is impossible . So solution $(8,10,0,3)$ does not exist. (iii) Let $\mathrm{B}$ be a blocking set having the solution $(11,1,9,0)$. Since $\mathrm{c}>0$, let $\ell$ be a 3-secant. Now any two 3-secant must be intersect in a point of $B$. Since if two3-secant intersect in a point $\mathrm{p} \notin \mathrm{B}$, then $|\mathrm{B}| \geq 2 * 3+3 * 1=9$ which is impossible. On every $p \in B$ there are at most three 3 -secants passing through $\mathrm{p}$.

Now since $T_{3}=9$ then the remaining eight 3 -secants pass through the three points of $\ell \cap \mathrm{B}$, So we have a point of $\ell \cap \mathrm{B}$ with at least four 3 -secants, and that is impossible. Hence $(11,1,9,0)$ does not exist.

The following lemma gives crucial information on the structure of such a blocking set. This lemma was proved by Ga'cs [5] using the Re'dei-polynomial [10]. It will enable us to eliminate the existence of such minimal blocking sets. 
Lemma( 3.2.6): Ga'cs [5]. In PG(2,q)let B be a minimal blocking set of size

$\mathrm{q}+\mathrm{k}$, and suppose there is a line 1 intersecting $\mathrm{B}$ in exactly $\mathrm{k}-1$ points. Then there is a point $\mathrm{O} \notin \mathrm{B}$ such that every line joining $\mathrm{O}$ to a point of 11 $B$ contains two points of $B$. Hence $k \geq(q+3) / 2$.

The only possibility for a minimal blocking set of size eight in $\mathrm{PG}(2,4)$ that remains is a blocking set containing a 4-secant ; in other words a blocking set of Rédei -type.

Theorem( 3.2.7). There is a minimal blocking set of size eight of Rédei -type in $\mathrm{PG}(2,4)$.

Proof. Let $(x, y, z)$ denote the coordinates of a projective point. Let 1 be a 3 -secant to $\mathrm{B}$. Let 1 be the line at infinity $(\mathrm{z}=0)$ of the corresponding affine plane, and let $\{\mathrm{P} 1, \mathrm{P} 2\}=1 \backslash \mathrm{B}$. By Lemma (4.2.6), there is an affine point $\mathrm{O} \notin \mathrm{B}$ for which the lines $\mathrm{OPi}, \mathrm{i}=1,2$, are bisecants . These lines contain four affine points of $\mathrm{B}$. Let $\mathrm{U}$ be the 5 th affine point of $\mathrm{B} \backslash$. Since the points Pi only lie on bisecant and three tangents, the lines UPi are tangents for $\mathrm{i}=1,2$.

Furthermore, the line $\mathrm{OU}$ is a line passing through a point of $\mathrm{B} \cap 1$.

Let $\mathrm{P}_{1}=(1,0,0), \mathrm{P}_{2}=(0,1,0)$, Assume OU passing through $(1,1,0)$. Since no three of $\left\{\mathrm{P}_{1}, \mathrm{P}_{2}, \mathrm{O}, \mathrm{U}\right\}$ are collinear we can consider $\mathrm{O}=(0,0,1)$ ,U=(1,1,1).

Consider now the affine plane $P G(2,4) \backslash 1$. Let $B^{\prime}=B \backslash(1 \cup\{U\})$. Then two points of $\mathrm{B}^{\prime}$ lie on $\mathrm{X}=0$, two on $\mathrm{Y}=0$. Since these are the lines $\mathrm{OPi}, \mathrm{i}=1,2$. Moreover, on every horizontal line $\mathrm{Y}=\mathrm{k}$, vertical line $X=k$, and on every line there is one point of $B$,in particular on line $\mathrm{y}=1, \mathrm{x}=1, \mathrm{y}=\mathrm{x}$ which all passing through $\mathrm{U}$ there is no point of $\mathrm{B}^{\prime}$, Let the points of $\mathrm{AG}(2,4)$ be .

$$
\begin{aligned}
& (0,0),(0,1),(0, w),\left(0, w^{2}\right) \\
& (1,0),(1,1),(1, w),\left(1, w^{2}\right) \\
& (w, 0),(w, 1),(w, w),\left(w, w^{2}\right) \\
& \left(w^{2}, 0\right),\left(w^{2}, 1\right),\left(w^{2}, w\right),\left(w^{2}, w^{2}\right)
\end{aligned}
$$

On $\mathrm{OP}_{1} ; \mathrm{Y}=0$, the remaining two points which are not belonging to any line through $\mathrm{U}$ are $\mathrm{l}_{1}=\left\{(\mathrm{w}, 0),\left(\mathrm{w}^{2}, 0\right)\right\}$.

On $\mathrm{OP}_{2} ; \mathrm{X}=0$, the remaining two points which are not belonging to any line through $U$ are $l_{2}=\left\{(0, w),\left(0, w^{2}\right)\right\}$. Chosen the point $(0, w),($ $\left.0, \mathrm{w}^{2}\right)$, on $\mathrm{x}=0$ does not eliminate any points of $1_{1}$ also chosen $(\mathrm{w}, 0)$ , $\left(\mathrm{W}^{2}, 0\right)$ does not eliminate any points of $1_{2}$; in B.So the set $\mathrm{B} \cap 1 \cup\{$ $\left.(\mathrm{w}, 0,1),\left(\mathrm{w}^{2}, 0,1\right),(0, \mathrm{w}, 1),\left(0, \mathrm{w}^{2}, 1\right),(1,1,1)\right\}=$ $\left\{(1,1,0),(\mathrm{w}, 1,0),\left(\mathrm{w}^{2}, 1,0\right),(\mathrm{w}, 0,1),\left(\mathrm{w}^{2}, 0,1\right),(0, \mathrm{w}, 1),\left(0, \mathrm{w}^{2}, 1\right),(1,1,1)\right\}$ form a minimal blocking set of Rédei-type. 


\section{The Lower Bounds of Eight and Fourth ...}

\subsection{Minimal Blocking sets in $\operatorname{PG}(2,5)$ :}

The following lemmas give the properties of minimal blocking sets of size ten.

Lemma( 3.3.1): Every blocking set of size ten in $\mathrm{PG}(2,5)$ has at least four points on a line.

Proof. Suppose there are only 1-,2-, and 3-secants.let the number of them be denoted by a, b , c, resp. Then the following equations must hold by standard counting arguments.

$$
\begin{aligned}
& a+b+c=31 \\
& a+2 b+3 c=60 \\
& 2 b+6 c=90 \ldots
\end{aligned}
$$

From these equations, we get $b=-3$ which is impossible.

Lemma (3.3.2): There are at most three 4-secant through any point of B. Proof. Every two 4-secants to B are intersected in a point on $\mathrm{B}$, if two 4-secant interse in $\mathrm{p} \quad \mathrm{B}$ then $|\mathrm{B}| \geq 2 * 4+4=12$, which is impossible , New assume there are four 4-secants through a point $p \in B$, then $|B| \geq 3 * 4+1=13$ and that is impossible. So through every point of $B$ there are at most three 4 -secants.

Lemma( 3.3.3): There are no minimal blocking sets of size ten with 4secant but no 3-secant .

Proof. Suppose there are only 1-,2-,and 4- secants. Let the numbers of them be denoted by a,b,d,resp. Then the following equations must hold by standard counting arguments.

$$
\begin{aligned}
& a+b+d=31 \\
& a+2 b+4 d=60 \\
& 2 b+12 d=90 \ldots
\end{aligned}
$$

From these equations we get $3 d=16$ which is not possible for 3 does not divide 16 .

Lemma (3.3.4): If B has no 2-secant, then B has at least one 4-secant Proof. Suppose there are only 1-,3-,and 4- secants. Let the number of them be denoted by a, c ,d .Then the following equations must hold by standard counting arguments.

$$
\begin{aligned}
& a+c+d=31 \\
& a+3 c+4 d=60 \\
& 6 c+12 d=90
\end{aligned}
$$

From these equations we get $\mathrm{d}=1$.

It is easy to prove.

Lemma( 3.3.5) : Let $1_{1}$ be a 4-secant to $B$ and $l_{2}$ be a 3 -secant to $B$ then $1_{1} \cap l_{2}$ be a point in $\mathrm{B}$. 
Lemma( 3.3.6) : Let 1 be a 4-secant to $B$ then through any point of $1 \cap \mathrm{B}$ there is at most three 3 -secant.

Proof. Let $\mathrm{p}$ be a point of $1 \cap \mathrm{B}$ and assume there are four 3-secant through $p$,then $|B| \geq 4+2 * 4=12$ which contradict the size of $B$.

Theorem (3.3.7): Let B have at most four points on a line. Let the number of 1-, 2-, 3- and 4-secants be denoted by a, b, c, d resp. Then these numbers satisfy one of the following possibilities:

\begin{tabular}{|c|c|c|c|c|}
\hline $\mathrm{a}$ & $\mathrm{b}$ & $\mathrm{c}$ & $\mathrm{d}$ & Possibilities \\
\hline \hline 13 & 12 & 1 & 5 & ( i ) \\
\hline 14 & 9 & 4 & 4 & ( ii ) \\
\hline 15 & 6 & 7 & 3 & ( iii ) \\
\hline 16 & 3 & 10 & 2 & ( iv ) \\
\hline 17 & 0 & 13 & 1 & (v) \\
\hline
\end{tabular}

Proof. The standard counting arguments give:

$a+b+c+d=31$

$\mathrm{a}+2 \mathrm{~b}+3 \mathrm{c}+4 \mathrm{~d}=60$

$2 b+6 c+12 d=90$

From these we can deduce

$\mathrm{a}=18-\mathrm{d}$;

$\mathrm{b}=-3+3 \mathrm{~d}$

$\mathrm{c}=16-3 \mathrm{~d}$

Since $c \geq 0$, we get $d \leq 5$.

Theorem(3.3.8): The solution $(17,0,13,1)$ of Theorem(3.3.7)does not exist.

Proof. Let 1 be a 4-secant.Since there are thirteen 3 -secants, and since every 3 -secant must intersect the 4 -secant 1 in a point in $\mathrm{B}$, so we have a point $\mathrm{p}$ in $\mathrm{B}$ Through which pass at least four 3 -secants, and that contradicts to Lemma (3.3.6).

\subsubsection{Minimal blocking sets of size ten with at most 4-} secants:

We find an example of minimal blocking sets of size ten with ten points.

Example (3.3.10) :In $\mathrm{PG}(2,5)$ the set of the points $\{(1,2,0),(1,-$ $1,0),(0,1,-1)$, $(1,-2,0),(0,1,-2),(1,1,2),(1,1,0),(1,1,1),(1,0,-1),(1,0,-2)\}$ is minimal blocking set with $\mathrm{T}_{1}=14, \mathrm{~T}_{2}=9, \mathrm{~T}_{3}=4, \mathrm{~T}_{4}=4, \mathrm{~T}_{5}=0$. 


\section{The Lower Bounds of Eight and Fourth ...}

\subsubsection{Minimal blocking sets of size ten with 5-secants:}

The following theorems prove that the existence of minimal blocking sets of size ten, $\mathrm{T}_{5}>0, \mathrm{~T}_{4} \neq 0$.

Theorem(3.3.12): Let $\mathrm{B}$ have at most 5 points on a line. Let the numbers of 1-, 2-, 3-,4- and 5-secants be denoted by a, b, c, d,e resp . Then these numbers satisfy one of the following possibilities:

\begin{tabular}{|c|c|c|c|c|c|}
\hline $\mathrm{a}$ & $\mathrm{b}$ & $\mathrm{c}$ & $\mathrm{d}$ & $\mathrm{e}$ & Possibilities \\
\hline 11 & 16 & 1 & 1 & 2 & (i) \\
\hline \hline 12 & 13 & 4 & 0 & 2 & (ii) \\
\hline \hline 12 & 14 & 1 & 3 & 1 & (iii) \\
\hline 13 & 11 & 4 & 2 & 1 & (iv) \\
\hline 14 & 8 & 7 & 1 & 1 & (v) \\
\hline \hline 15 & 5 & 10 & 0 & 1 & (vi) \\
\hline
\end{tabular}

Proof. The standard counting arguments give:

$\mathrm{a}+\mathrm{b}+\mathrm{c}+\mathrm{d}+\mathrm{e}=31$

$a+2 b+3 c+4 d+5 e=60$

$2 b+6 c+12 d+20 e=90$

From these we can deduce

$\mathrm{c}=-3 \mathrm{~b}-6 \mathrm{a}+115$

$\mathrm{d}=8 \mathrm{a}+3 \mathrm{~b}-135$

$\mathrm{e}=-3 \mathrm{a}-\mathrm{b}+51$

Since $\mathrm{d} \geq 0$, we get $\mathrm{e} \leq 2$.

Theorem(3.3.13): There are Rédei -type minimal blocking sets of size ten in $\operatorname{PG}(2,5)$.

Proof. Let $B$ be a blocking set with $e>0, d \neq 0$. Let 1 be a 4-secant to $B$. and assume 1 is the line at infinity of the corresponding affine plane $(\mathrm{z}=0)$, and let $\{\mathrm{P} 1, \mathrm{P} 2\}$ be the points $1 \backslash \mathrm{B}$. By Lemma (3.2.6), there is a point $\mathrm{O} \notin \mathrm{B}$ such that $\mathrm{OP}_{1}, \mathrm{OP}_{2}$ are bisecants to $\mathrm{B}$. Let $\mathrm{U}_{1}, \mathrm{U}_{2}$ be the remaining points of $\mathrm{B}$, and assume $\mathrm{P}_{1}=(1,0,0), \quad \mathrm{P}_{2}$ $=(0,1,0), \mathrm{O}=(0,0,1), \mathrm{U}_{1}=(1,1,1)$. Nowthe affine lines joining $\mathrm{OP}_{1}, \mathrm{OP}_{2}$ are $\mathrm{y}=0, \mathrm{x}=0$. The lines joining $\mathrm{P}_{1} \mathrm{U}_{1}, \mathrm{P}_{2} \mathrm{U}_{2}$ either tangent to $\mathrm{B}$ or pass through $\mathrm{U}_{2}$.On $\mathrm{OP}_{1} ; \mathrm{Y}=0$, we need to select two points of the set $1_{1=}\{(1,0,-2),(1,0,-1),(1,0,2)\}$, and on $\mathrm{OP}_{2} ; \mathrm{X}=0$, we need to select two points of the set $1_{2=}\{(0,1,-2),(0,1,-1),(0,1,2)\}$. Choose $(1,0,-2),(1,0,-1)$ from $1_{1}$, and $(0,1,-2),(0,1,2)$, and $\mathrm{U}_{2}=(1,-2,2)$ with the four points at $\mathrm{z}=0$ in $\mathrm{B}$ and $\mathrm{U}_{1}$, these ten points form minimal blocking set. 


\section{References}

[1] Ball ,S. (1996),"Multiple blocking sets and arcs in finite planes”, J. London Math. Soc. 54, 581-593.

[2] Ball ,S. and Hirschfeld, J.W.P. (2005),"Bounds on(n, r)-arcs and their application to linear codes”, J. Geom,1-11.

[3] Bruen ,A. A. (1986), "Arcs and multiple blocking sets", Combinatorica, Symposia Mathematica 28 , Academic Press, 15-29.

[4] Di Paola, J. (1969), “ On minimum blocking coalitions in small projective plane games”, SIAM J. Appl. Math., 17, 378-392.

[5] Ga'cs, A. (1997), “The Re'dei Method Applied to Finite Geometry”, Ph.D. Thesis , Eo"tvo"s Lora'nd University, Budapest(Hungary).

[6] Hirschfeld, J.W.P, (1979),“ Projective Geometries over Finite Fields”, Oxford University Press, Oxford.

[7] Hirschfeld ,J.W.P. and Storme ,L. ( 2001), “ The packing problem in statistics, coding theory and finite projective spaces", update 2001, in: Finite Geometries, Developments in Mathematics 3, Kluwer, 201-246.

[8] Kasm ,N.Y. (2006), "Lower bounds of t-fold blocking sets and construction of $(\mathrm{k}, \mathrm{n})$-arcs in the desargusian plane $\mathrm{PG}(2, \mathrm{q})$ ",

Ph.D.Thesis,University of Mosul.

[9] Raymond ,H.(1982), "Some problems conserning (k,n)-arcs in finite projective planes", Rend. Sem. Mat. Brescia 7,367-383.

[10] Re'dei , L. (1973), "Lacunary Polynomials over finite fields ", Birkhäuser Verlag, Basel.

[11] Richardson,M. (1956), “ On Finite projective games”, Proc. Amer. Math. Soc., 7, 458-465.

[12] Yasin ,A .L.(1986), "Cubic arcs in projective plane of order eight",

$\mathrm{Ph}$. D. Thesis, University of Sussex, England. 